\title{
Aspiration pneumonia in the child with DiGeorge syndrome -A case report-
}

\author{
Ji-Young Lee, and Yun-Joung Han \\ Department of Anesthesiology and Pain Medicine, St. Mary's Hospital, The Catholic University of Korea College of Medicine, Seoul, Korea
}

DiGeorge syndrome is associated with a chromosome 22q11.2 deletion and manifests with variable clinical findings. Aspiration pneumonia can be a perioperative complication of great concern in this syndrome. In this report, we present a case of a 16-month old child with DiGeorge syndrome undergoing cranioplasty. He developed perioperative aspiration pneumonia but was managed successfully. (Korean J Anesthesiol 2011; 60: 449-452)

Key Words: Aspiration pneumonia, DiGeorge syndrome.

Chromosome 22q 11.2 deletion syndrome is relatively common - the incidence is $1 / 3000$. The same deletion can result in various phenotypic presentations such as DiGeorge syndrome, CATCH-22 syndrome, and velocardiofacial syndrome [1,2]. DiGeorge syndrome presents with micrognathia, congenital heart disease, hypocalcemia, seizure, and cellular immunodeficiency because of anomalies in the thymus, parathyroid and great vessels [3]. Anesthesiologists should focus on the possibilities of difficult intubation due to facial anomalies and endobronchial intubation due to a short trachea. Preoperative cardiac evaluation, perioperative calcium monitoring, and use of radiologically treated blood products should also be considered [4].

Several cases of aspiration during the clinical course in children with DiGeorge syndrome have been reported [3], but there are few reports regarding the incidence and importance of aspiration pneumonia in the perioperative period. We recently had to deal with aspiration pneumonia in a child with DiGeorge syndrome undergoing elective surgery.

\section{Case Report}

A 16-month old, $10 \mathrm{~kg}$ male child was scheduled for a cranioplasty with duroplasty due to craniosynostosis. He was born at full-term with a birth weight of $3.88 \mathrm{~kg}$. He received palatoplasty 14 month after birth, and was diagnosed as having DiGeorge syndrome by a genetic study after the surgery. He had no history of serum calcium level-related seizures, no feeding difficulties, and no other neuromuscular deformity. An elective cranioplasty was postponed twice due to intermittent high fever. The pediatrician treated the patient for upper respiratory tract infection on each of those occasions.

During preoperative evaluation, there was no specific finding on EKG or echocardiography, no definite anomaly of the great vessels and trachea on lung CT, and blood calcium levels were normal. Apical herniation of the right lung and thymic agenesis

Received: July 15, 2010. Revised: 1st, August 6, 2010; 2nd, November 15, 2010; 3rd, December 27, 2010. Accepted: December 27, 2010.

Corresponding author: Ji-Young Lee, M.D., Department of Anesthesiology and Pain Medicine, St. Mary's Hospital, The Catholic University of Korea College of Medicine, 62, Yeouido-dong, Yeongdeungpo-gu, Seoul 150-713, Korea. Tel: 82-2-3779-1944, Fax: 82-2-783-0368, E-mail: leeji@catholic.ac.kr (c) This is an open-access article distributed under the terms of the Creative Commons Attribution Non-Commercial License (http:// creativecommons.org/licenses/by-nc/3.0/), which permits unrestricted non-commercial use, distribution, and reproduction in any medium, provided the original work is properly cited. 
on chest radiography was found (Fig. 1). Craniosynostosis, cleft palate and thymic agenesis were relevant to DiGeorge syndrome, but serum total and ionized calcium levels were normal.

Anesthesia was induced with ketamine (15 mg) with glycopyrrolate $(0.05 \mathrm{mg})$ followed by succinylcholine (15 mg). We intubated the trachea with an internal diameter 3.5 $\mathrm{mm}$ endotracheal tube with cuff, and inflated the balloon. Anesthesia was maintained with sevoflurane 2.0\% - $\mathrm{N}_{2} \mathrm{O} 50 \%$ - $\mathrm{O}_{2} 50 \%$ and vecuronium bromide. End tidal carbon dioxide concentration was $39-46 \mathrm{mmHg}$ at $120 \mathrm{ml}$ of tidal volume and 16-18 mmHg of peak inspiratory pressures.

After 1 hour of intubation, and during central venous catheterization via the subclavian vein, the patient's oxygen saturation decreased to $60 \%$. We suspected this event was due to an airway problem caused by head and neck movement, or to a traumatized lung due to a central line catheterization. We stopped the catheterization procedure and ventilated the patient manually with $100 \%$ oxygen. During manual ventilation, we found that the airway had no obstructions, and we heard coarse lung sounds from both lungs. The patient recovered to $100 \%$ oxygen saturation within 3 minutes. We replaced nitrous oxide with room air, and a tracheobronchial toilette was performed to check the patency of the tube and to remove secretions. The tube was intact, and only a small amount of viscous secretions was suctioned in spite of coarse breathing sounds. To rule out pneumothorax, we did chest radiography and confirmed acute infiltration in both upper lung fields (Fig. 2). Since vital signs were stable, and the reservoir bag was compliant during manual ventilation, the operation continued. A central line catheterization was done via the jugular vein

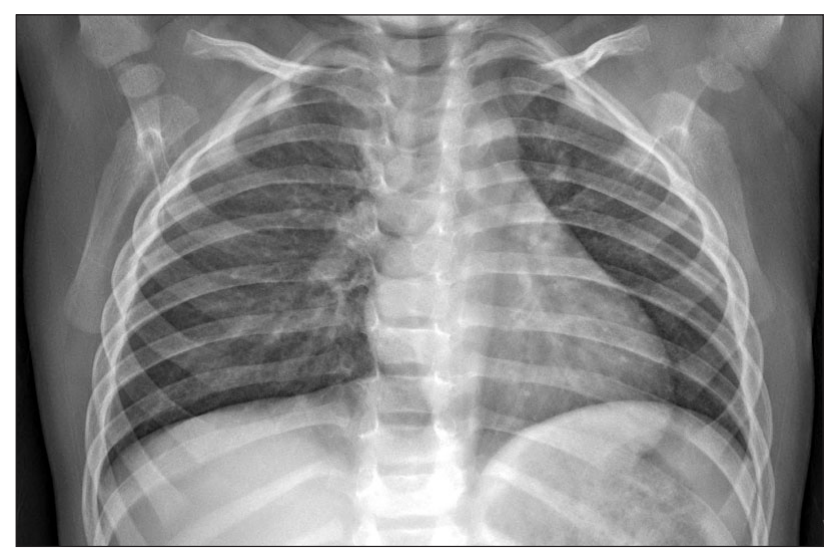

Fig. 1. A preoperative chest PA on the day before surgery shows a narrowed superior mediastinum suggesting thymic agenesis, apical herniation of the right lung and a resultant left sided buckling of the adjacent trachea air column. instead of the subclavian vein. Cranioplasty and duroplasty were done for metopic synostosis, which took 7 hours. During the operation, vital signs were stable. Central venous pressure remained at $3 \mathrm{mmHg}$ to $7 \mathrm{mmHg}$, systolic blood pressure from $80 \mathrm{mmHg}$ to $100 \mathrm{mmHg}$, and heart rate from 105 beats/min to 130 beats $/ \mathrm{min}$. The results of arterial blood gas analysis were acceptable. We made a considerable effort to avoid hyperventilation in order to eliminate the possibility of hypocalcemia. We transfused $200 \mathrm{ml}$ of packed RBC and $100 \mathrm{ml}$ of fresh frozen plasma.

At the end of the surgery, as the patient started to breathe spontaneously, a huge amount of secretions gushed out of the endotracheal tube. They were slightly yellow, highly viscous, but not frothy. Since there was a possibility of hypervolemia caused by increased venous return accompanied by recovery of the sympathetic system, we injected $6 \mathrm{mg}$ of furosemide. We suspected a pathologic lung condition other than transudative pulmonary edema due to the characteristics of the secretion. Chest radiography was checked again. Radiologists reported an atelectasis caused by pulmonary aspiration in both upper lung fields, dominant on the right side. The patient revealed a large amount of secretions and relative hypoxemia. $\mathrm{PaO}_{2}$ was 105 mmHg. We transported the patient to the ICU after injecting him with 2 mg of midazolam, and continued mechanical ventilation with PEEP. Treatments with antibiotics, mucolytics, $\mathrm{H}_{2}$-blocking agents, and chest physiotherapy with postural drainage were started.

The patient improved symptomatically, and was extubated at postoperative day 3 . There were multiple linear and segmental atelectasis on the dependent portion of the right upper and lower lobes on the chest CT (Fig. 3). The patient's pulmonary

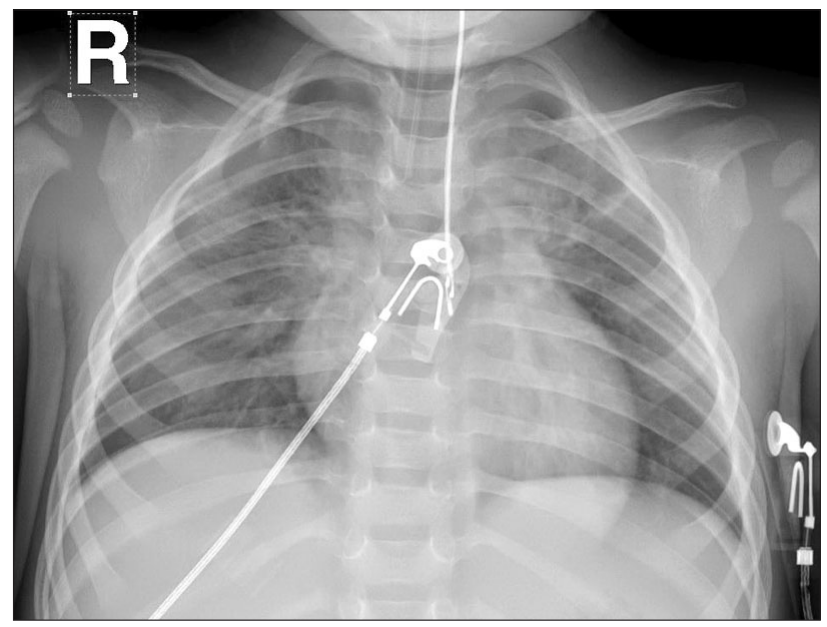

Fig. 2. Intraoperative chest AP film showing newly developed streaky and patch opacities in both upper lung fields. ETT above the carina is also shown. 


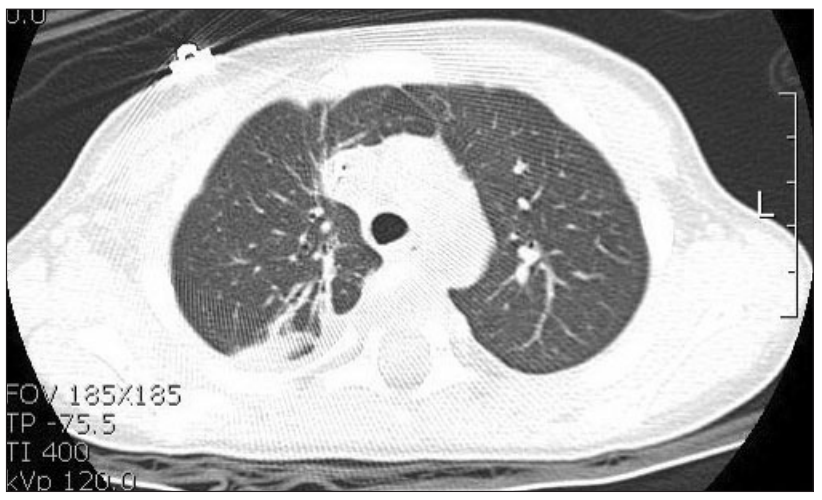

Fig. 3. Multiple remnant subsegmental atelectasis is shown in dependent portion of RUL on chest CT at postoperative day 3 .

condition was improving, but he was intermittently febrile in spite of continuous antibiotic therapy. The fever fluctuated from 37.5 to $39.2^{\circ} \mathrm{C}$ for 3 weeks. Acinetobacter baumani was grown in culture from blood and indwelling catheter tips. After continued susceptible antibiotic therapy, the patient was discharged at postoperative day 24 . He was recommended for a further study of his infection and an immunologic study.

\section{Discussion}

Hemizygous 22q11.2 deletion syndrome has been called several names depending on the variable combinations of symptoms such as DiGeorge syndrome, velocardiofacial syndrome, or CATCH 22 syndrome [1]. DiGeorge syndrome manifests with craniofacial dysmorphism, and defects in the heart, parathyroid, and thymus. Immunologic deficiencies secondary to thymic agenesis or hypoplasia, and hypocalcemia secondary to hypoparathyroidism are the main manifestations with congenital cardiac anomalies [2,3]. From the anesthesiologist's point of view, short trachea [5], hypocalcemic seizure [6], and postoperative tachycardia after multiple dental extractions using local epinephrine were commented [7]. Gastric regurgitation or aspiration was rarely mentioned as one of the symptoms. Therefore, prevention of aspiration was not emphasized. In infants and children, mortality and morbidity from aspiration pneumonia are rare for elective surgical procedures. However, aspiration pneumonia could be serious when it occurs in an immunocompromised patient such as one with DiGeorge syndrome. To prevent aspiration, it is very important to evaluate the patient thoroughly, and administer a nonparticulate antacid, metoclopramide, or $\mathrm{H}_{2}$-blocking agents [8].

Markert et al. [3] reviewed 8 patients with complete DiGeorge syndrome, and reported that 2 patients died from gastroesophageal reflux with aspiration. More surprisingly, all eight patients presented with gastroesophageal reflux. It was also suggested that this syndrome presented with pharyngeal and esophageal dysmotility [2]. Gastroesophageal reflux, and pharyngeal and esophageal dysmotility may contribute to development of aspiration. This fact indicates the need to focus on the possibility and on prevention of aspiration in patients with DiGeorge syndrome.

Chegar et al. [9] examined velum, pharynx and larynx in patients with velocardiofacial syndrome, which has the same 22q 11.2 deletion syndrome as DiGeorge syndrome. They reported asymmetric development of the pharynx and larynx. These developmental abnormalities may also increase the risk of aspiration and airway obstruction.

In our case, aspiration may have occurred during the induction period. Preoperative chest x-ray on the day before surgery did not show any abnormality in both lung fields. Symptoms and abnormal x-ray findings appeared within 2 hours of induction, which is very relevant to aspiration pneumonia. Clinical signs, such as coughing, bucking, and incomplete paralysis supporting aspiration did not occur. It is known that hospital-acquired pneumonia occurs as a consequence of frequent silent aspirations of nasal, throat, and periodontal secretions [10]. Besides silent aspiration, succinylcholine could be a causative factor, even with sufficient fasting time before the operation, and complete paralysis. The reasons for choosing succinylcholine over non-depolarizing muscle relaxants was previous palatoplasty and the dysmorphic facial features of DiGeorge syndrome. The surgery narrowed the oral cavity, and we could not guarantee successful intubation. Aspiration could have occurred even if we used non-depolarizing relaxants.

Though the clinical course was typical for aspiration pneumonia, we could not detect the cause for the large amount of thick secretions. Sudden gushing out of secretions at the end of the anesthesia made us confuse the situation with pulmonary edema. During surgery, the airway pressure was not too high. However, the coarse breathing sound after several tracheobronchial suctions could lead to the possibility of huge secretions in the bronchiolar tree and pulmonary parenchyma. Also, subclinical pulmonary edema could have been superimposed on aspiration pneumonia without frothy secretion.

Because of postoperative intermittent high fever and bacterial growth in the blood, we assumed immunodeficiency in DiGeorge syndrome to be the cause. Immunoglobulin G, A, and M were subnormal. A further immunologic study was definitely essential, but the parents refused.

DiGeorge syndrome is associated with highly variable clinical manifestations - over 180 features. Gastroesophageal reflux and aspiration could be amongst them. In conclusion, we suggest active prevention of aspiration in patients with DiGeorge syndrome. 


\section{References}

1. Sullivan KE. Chromosome 22q11.2 deletion syndrome: DiGeorge syndrome/Velocardiofacial Syndrome. Immunol Allergy Clin North Am 2008; 28: 353-66.

2. Baum VC, O'Flaherty JE. Anesthesia for genetic, metabolic, and dysmorphic syndromes of childhood. 2nd ed. Philadelphia, Lippincott Williams \& Wilkins, a Wolters Kluwer business. 2007, pp103-4.

3. Markert ML, Hummell DS, Rosenblatt HM, Schiff SE, Harville TO, Williams LW, et al. Complete DiGeorge syndrome: persistence of profound immunodeficiency. J Pediatr 1998; 132: 15-21.

4. Cladis FP. Index of syndromes and their pediatric anesthetic implications. In: Smith's Anesthesia for Infants and Children. 7th ed. Edited by Motoyama EK, Davis PJ: Philadelphia, Mosby Inc. 2006, p 1216.

5. Wells AL, Wells TR, Landing BH, Cruz B. Galvis DA. Short trachea, a hazard in tracheal intubation of neonates and infants: syndromal associations. Anesthesiology 1989; 71: 367-73.

6. Singh VP, Agarwal RC, Sanyal S, Waghray MR, Luthra ML, Borcar JM. Anesthesia for DiGeorge's syndrome. J Cardiothorac Vasc Anesth 1997; 11: 811.

7. Passariello M, Perkins R. Unexpected postoperative tachycardia in a patient with 22q11 deletion syndrome after multiple dental extractions. Paediatr Anaesth 2005 ; 15: 1145-6.

8. Ghazal EA, Mason LJ, Cote CJ. Preoperative Evaluation, premedication, and induction of anesthesia. In: A Practice of Anesthesia for Infants and Children. 4th ed. Edited by Cote CJ, Lerman J, Todres ID: Philadelphia, Saunders Elsevier. 2009, pp 37-69.

9. Chegar BE, Tatum SA 3rd, Marrinan E, Shprintzen RJ. Upper airway asymmetry in velo-cardio-facial syndrome. Int J Pediatr Otorhinolaryngol 2006 ; 70: 1375-81.

10. Japanese Respiratory Society. Aspiration pneumonia. Respirology 2009; 14: S59-64. 